\title{
Compact pyroelectric detectors based on micro-machined chip carriers
}

\author{
A.Günther ${ }^{1}$ and M.Ebermann ${ }^{1}$ \\ 1 InfraTec GmbH, Gostritzer Strasse 61-63, 01217 Dresden, Germany, \\ a.guenther@infratec.de
}

\begin{abstract}
:
A novel micro-machined chip carrier system for pyroelectric detectors was developed that integrates the sensor elements as well as the infrared filters into one assembly. Based on these chip carriers miniaturized multi-channel detectors with attractive electro-optical parameters especially for gas analysis measurements were engineered.
\end{abstract}

Key words: pyroelectric detector, miniaturized, multi-channel, gas analysis, high field of view

\section{Introduction}

Pyroelectric infrared detectors are a core component of measurement and control devices in industrial safety technology (e.g. monitoring of gas concentrations [1] and flame sensors in industrial plants or mines) as well as in medical sensor technology (e.g. anesthetic gas sensors or emergency medicine). Commercially available sensor models cover one- to four-channel detectors with transistor outline (TO) packages in various sizes. So far, InfraTec $\mathrm{GmbH}$ has offered dual-channel detectors in TO39 housing and four-channel ones in TO8. The progressive trend of sensor module miniaturization requires a stronger diminishment of the size of their single components, and a higher integration in smaller housings without a loss of performance. For this purpose a novel micro-machined chip carrier was developed. This chip carrier made from silicon facilitates a significant higher integration of the sensor elements and infrared filters. Therefore, it is possible to minimize the detector package size while the number of channels remains the same, or the number of channels can be increased while the housing remains the same size. Simultaneously, a large active area of the sensor elements and in addition a high field of view (FOV) can be realized. It is even possible to improve those parameters compared to previous solutions. Meanwhile the chip carriers are implemented in their own miniaturized detector family (Fig. 1).

\section{Conventional design of pyroelectric infrared detectors}

Pyroelectric detectors are classified as thermal sensors. Their functional principle is based on the pyroelectric effect of the applied sensor material. A change of the detector chip's temperature leads to a change of the materials surface charges that can be converted to an output voltage by an integrated amplifier [2]. Important application-relevant parameters are, besides the specific detectivity, the size of the active sensor element area as well as the FOV.
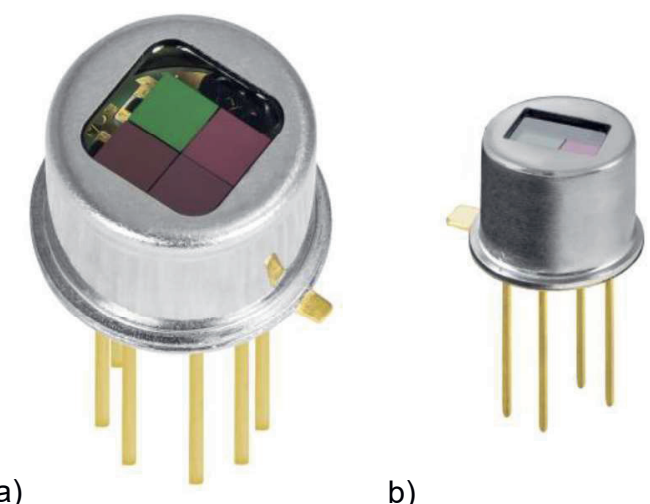

b)

Fig. 1: novel, high integrated multi-channel pyroelectric detectors a) four-channel detector LRM-254 b) dual-channel detector LRM-202

They decisively define the achievable level of the signal-to-noise (SNR) ratio. The applications mentioned above usually work in a frequency range from $1 \mathrm{~Hz}$ to $10 \mathrm{~Hz}$. In combination with pulsed thermal emitters the highest SNR can be achieved. Thus, there exists defined requirements on the detector's assembly and packaging techniques along with the hybrid integration of the sensor elements (e.g. made of $\mathrm{LiTaO}_{3}$ ). The size and the thickness of the elements as well as the thermal transfer to the housing and the ambient air should be optimized to realize a time constant 
in the range of 100 to $200 \mathrm{~ms}$. For several applications it is necessary to compensate for a change of the housing temperature. For this purpose, compensation elements are added and connected with the active elements in an antipolar manner (series or parallel compensation). The compensation elements are nearly identical to the active elements and their mounting technology but they are optically inactive. A typical detector structure of a pyroelectric, thermally compensated detector based on state of the art technology is schematically shown in Fig. 2. It has the following disadvantages:

- The active sensor elements are either directly mounted on the circuit board or on a special chip carrier. Compensation elements are added in the same plane and increase the required footprint.

- $\quad$ The FOV (for a given size of the detector elements) is limited by the opening in the cap (cap aperture). Due to the typical manufacturing and assembly tolerances no further optimization is possible.

- The application-specific infrared filters are adhesive bonded in the transistor cap. Therefore, the mostly sensitive filter coatings are directly exposed to environmental impacts. This could have a negative influence on their long-term stability.

- $\quad$ The size of the filters depend on the area of the cap apertures, which may result in higher material costs.

- To reduce optical crosstalk between neighboring channels it is necessary to add additional shields that need appropriate space.

\section{Novel modular chip carrier}

The solution to eliminate the aforementioned disadvantages and to optimize the relevant detector parameters entails a chip carrier that integrates the sensor elements as well as the infrared filters in a compact assembly into the detector housing. Fig. 3 shows the basic concept (a) and a micro-machined chip carrier without sensor elements for a dual-channel detector (b).

The concept has the following characteristics and advantages:

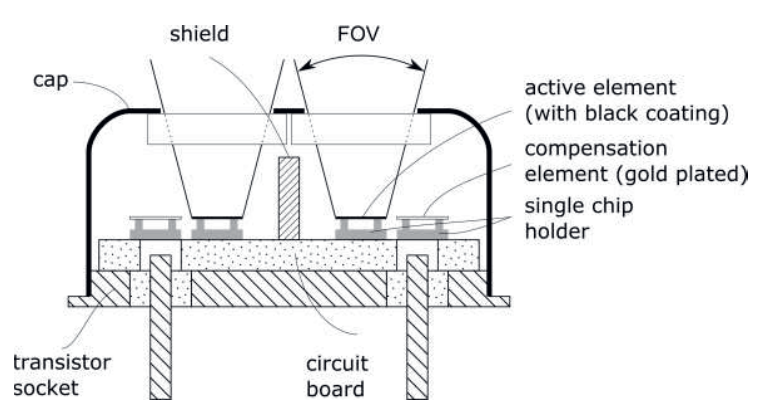

Fig. 2: Cross sectional view of the conventional detector structure

- cost-efficient micro-mechanical manufacturing process based on silicon, electrical conductivity due to metallization,

- $\quad$ small space requirement (compared to the active area of the pyroelectric sensor elements),

- $\quad$ enables the mounting of the infrared filters directly on top of the chip carrier and results in significant reduction of the filter size and material costs,

- modular concept, integration of several channels in one part, space saving configuration of compensation elements due to stacked structure,

- optimal thermal connection and effective optical shielding of the compensation elements,

- $\quad$ low heat dissipation due to minimal contact area for the sensor elements and optimized thickness of the ambient air layers (distance to the filters, the circuit board, and the chip carrier of the compensation elements),

- optimization of the FOV and effective suppression of (the) crosstalk between the channels.

Based on the benefits mentioned above compact, robust and cost efficient multi-channel detectors can be realized. Fig. 4 shows the schematic structure of a compensated fourchannel detector with the novel chip carrier in TO39 housing. In contrast to standard detectors, they are equipped with a broadband transmitting infrared window that is aligned with the inner infrared filters. 


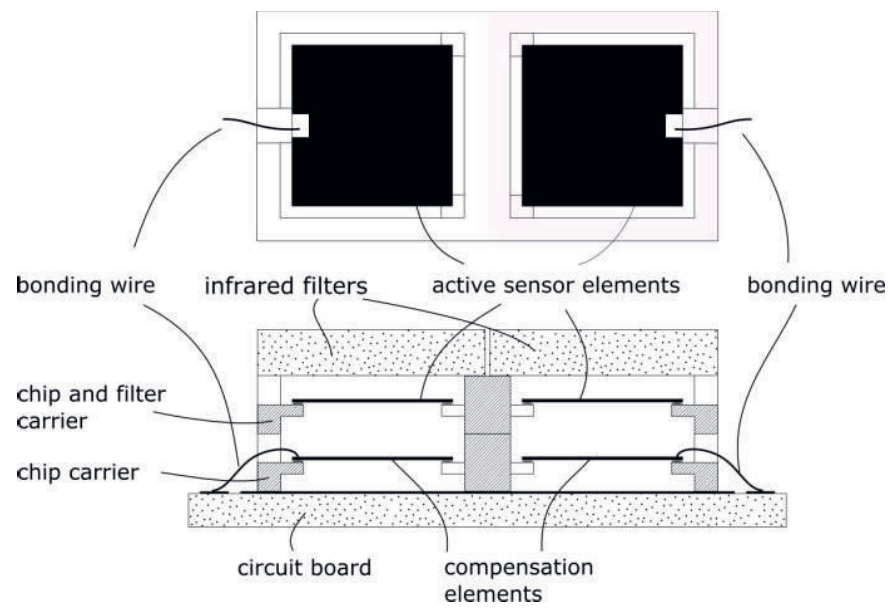

a)

Fig. 3: Basic concept of the novel chip carrier system (a) and a micro-machined chip carrier without sensor elements for a dual-channel detector (b).

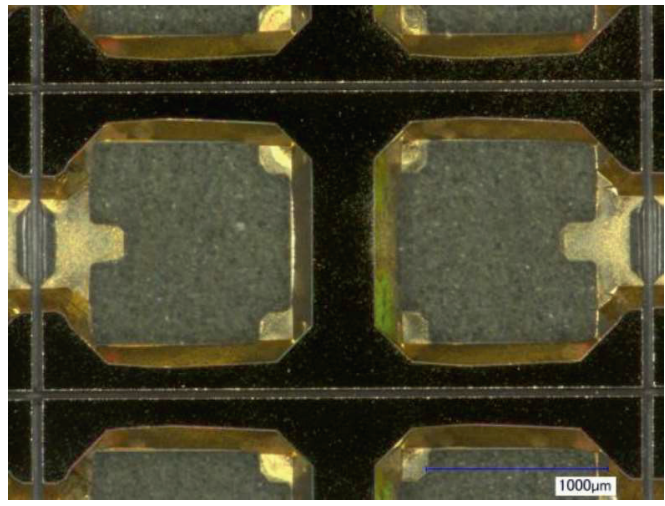

b)

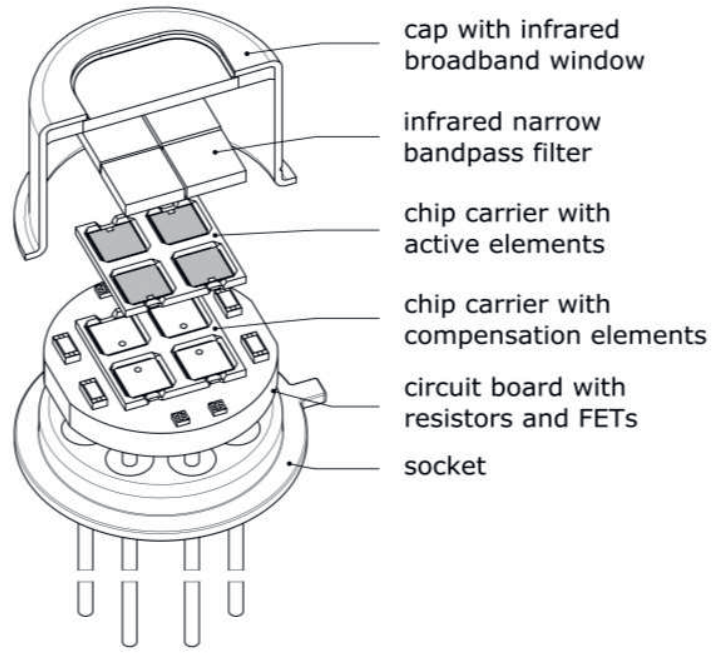

Fig. 4: Exploded view of a four-channel detector with novel micro-machined chip carrier (equivalent to Fig. 1)

\section{Comparison of detector characteristics}

The following section describes the advantages in more detail and quantitatively. The new developed detector LRM-254 is compared with standard detectors LIM-314 (four-channel, TO8) and LIM-222 (dual-channel, TO39) from InfraTec [3]. Fig. 5 shows all three detector types, Fig. 6 illustrates the different optical properties and important parameters are pointed out in Tab. 1.

High integration density

The significantly higher integration density of the LRM-254 becomes apparent in comparison with LIM-222. By having nearly the same active element area $\left(\sim 2 \mathrm{~mm}^{2}\right)$ and compensation elements, it is now possible to realize four instead of only two channels. So far that was only possible for TO8 housings (e.g. LIM-314) but with larger sensor elements having up to $4 \mathrm{~mm}^{2}$ active area.

Another advantage of the improved integration density is the reduction of the illuminated area and thus the utilization of space. Fig. 6 shows the degree of filling, which describes the ratio of the active sensor element area to the complete illuminated area. The degree of filling for the LRM-254 amounts $40 \%$ within a diameter of $4.7 \mathrm{~mm}$. The illumination occurs with a significantly lower loss compared to the standard detectors.

\section{Optimized Field of View}

The layout of the newly developed chip carriers was especially adapted to the requirements of a large FOV. This results in the small footprint of the carrier as well as the optimized distance between the mounted pyroelectric elements and the infrared filter above. As shown in Fig. 6, the LRM-254 has a 2.5x higher FOV than the LIM-222 with the same housing but double the number of channels.

\section{Protection of sensitive infrared filters}

As mentioned above, the newly developed detectors have a broadband infrared window, which is mounted into the cap. A protection of the sensitive infrared filters against environmental impacts results. Additional to that it is also possible to solder the window instead of adhesive bonding to protect the filters against penetrating humidity. 


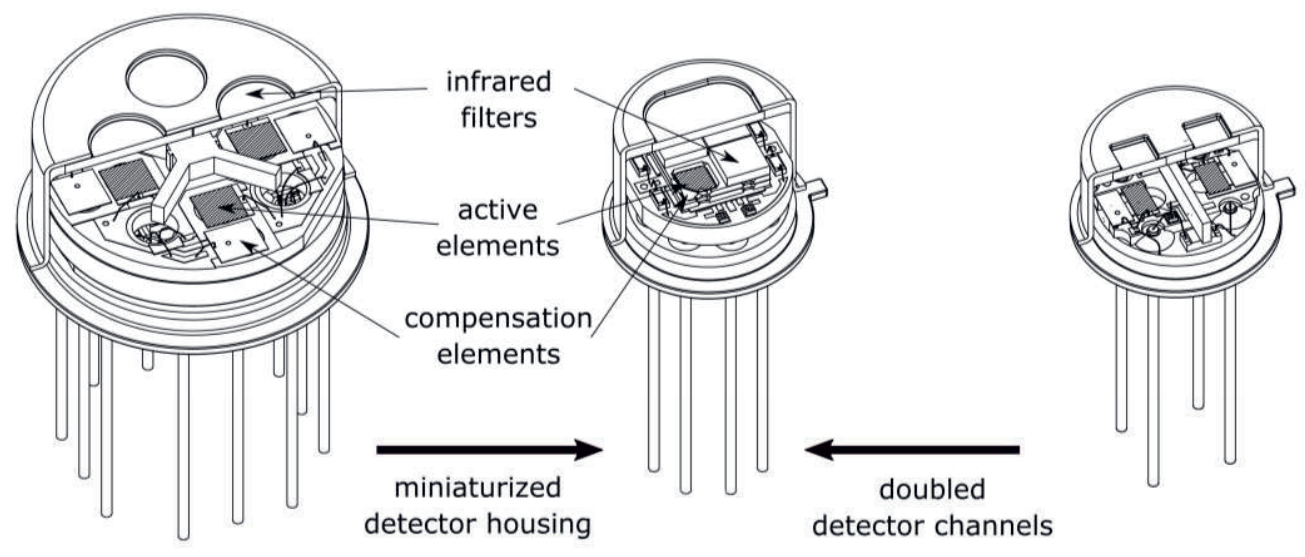

LIM-314

(TO8 socket, 4 channel)
LRM-254

(T039 socket, 4 channel)
LIM-222

(TO39 socket, 2 channel)

Fig. 5: Mounting system of standard detectors LIM-314 and LIM-222 compared to the new developed LRM-254
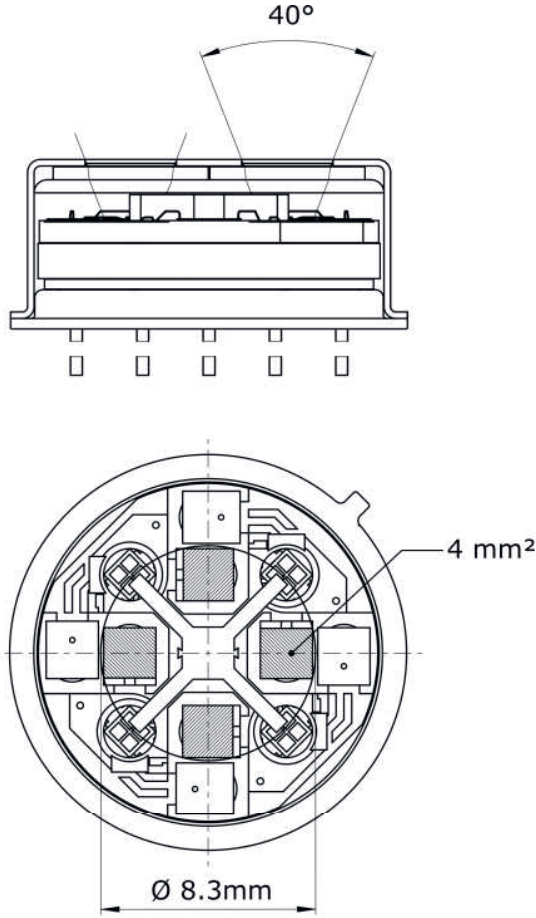

LIM-314
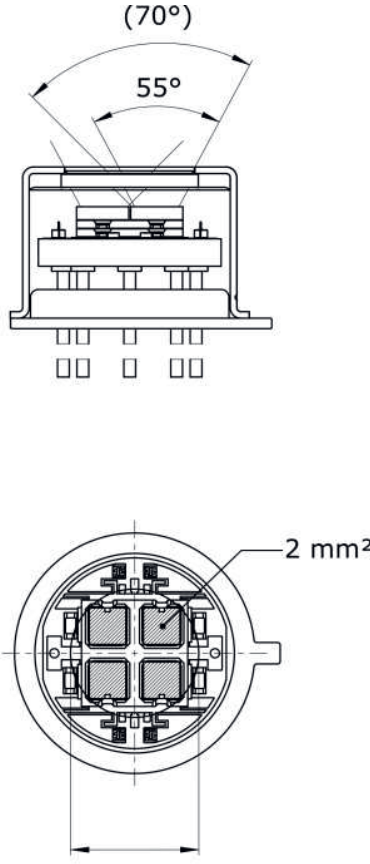

$\varnothing 4.7 \mathrm{~mm}$

LRM-254
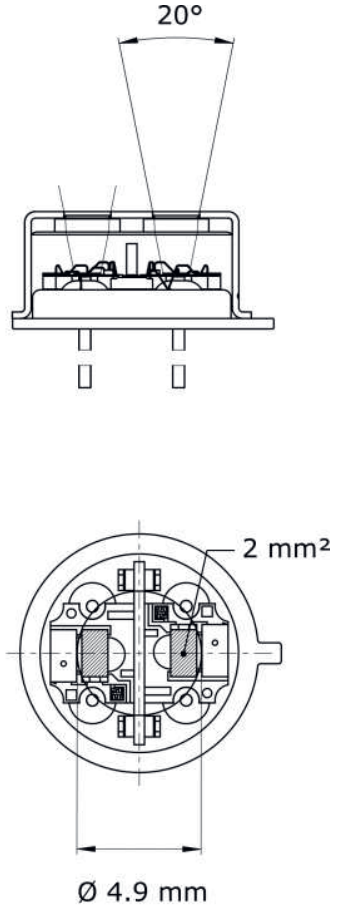

LIM-222

Fig. 6: Comparison of geometric-optical parameters for detectors LRM-254, LIM-222 and LIM-314 (substrate material for filters and windows: silicon, $0.5 \mathrm{~mm}$ thick)

Tab. 1: Comparison electro-optical properties standard detectors and LRM-254

\begin{tabular}{|c|c|c|c|c|c|c|c|}
\hline $\begin{array}{l}\text { Detector } \\
\text { type }\end{array}$ & Housing & Channels & $\begin{array}{l}\text { Element } \\
\text { size }\end{array}$ & $\begin{array}{l}\text { Diameter } \\
\text { (Spot size) }\end{array}$ & $\begin{array}{c}\text { Rel. } \\
\text { Sensitive } \\
\text { Area }\end{array}$ & FOV & $\begin{array}{c}D^{*} \\
{[\mathrm{~cm} \sqrt{ } \mathrm{Hz} / \mathrm{W}]^{1)}}\end{array}$ \\
\hline LIM-314 & TO-8 & 4 & $4 \mathrm{~mm}^{2}(\mathrm{x} 4)$ & $\varnothing 8,3 \mathrm{~mm}$ & $30 \%$ & $42^{\circ}$ & $3.5 \times 10^{8}$ \\
\hline LIM-222 & TO-39 & 2 & $2 \mathrm{~mm}^{2}(\mathrm{x} 2)$ & $\varnothing 4,9 \mathrm{~mm}$ & $41 \%$ & $20^{\circ}$ & $4.5 \times 10^{8}$ \\
\hline LRM-254 & TO-39 & 4 & $2 \mathrm{~mm}^{2}(\mathrm{x} 4)$ & $\varnothing 4,7 \mathrm{~mm}$ & $21 \%$ & $55^{\circ}$ & $4.5 \times 10^{8}$ \\
\hline
\end{tabular}




\section{Cost efficient manufacturing process}

The manufacturing of the chip carriers is done by a multistage micro-machining process. A silicon wafer is structured by photolithography and several etching processes. The electrical conductivity is realized by metallization of the wafer. Finally, the wafer is cut to separate chip carriers. Due to the modular design and depending on the required number of channels the carriers can be cut to the appropriate size.

It exists three different versions for the following $\mathrm{LiTaO}_{3}$ sensor element sizes: $(1.2 \times 0.8) \mathrm{mm}^{2}$, $(1.4 \times 1.4) \mathrm{mm}^{2}$ and $(2 \times 2) \mathrm{mm}^{2}$.

The detector assembly is done by automated die attach and wire bonding processes. The chip carriers can easily be taken from the wafer. Compared to standard detectors, the size of the most costly infrared filters is reduced, causing also a reduction of the material costs.

\section{Summary}

Based on a novel micro-machined chip carrier, a new family of miniaturized multi-channel detectors was developed. The concept expands the available degrees of freedom regarding the detector design and opportunities to optimize the detector. The newly introduced LRM-254 (TO39, four-channel) and LRM-202 (TO46, dual-channel) are very compact, thermally compensated (uncompensated also possible) voltage mode detectors. They especially meet the needs of space-saving measurement modules for gas analysis. Currently the LRM254 will be expanded by a single supply current mode type (LRM-284) with an integrated operational amplifier, whereby the integration density will be increased again.

\section{References}

[1] G. Wiegleb, Gasmesstechnik in Theorie und Praxis: Messgeräte, Sensoren, Anwendungen. Wiesbaden: Springer Vieweg, 2016.

[2] S.G. Porter, A Brief Guide to Pyroelectric Detectors, Ferroelectrics, Vol. 33, 193-206, (1981); doi:10.1080/00150198108008086

[3] InfraTec GmbH; Catalog 2013: Pyroelectric \& Multispectral Detectors, Dresden, InfraTec $\mathrm{GmbH}, 2013$,

http://www.infratec.de/de/sensorik.html. 